\title{
La Prévoyance dans l'Economie Italienne
}

\author{
par Giuseppe Petrilli *
}

\section{SUMMARY : SOCIAL POLICY IN THE ITALIAN ECONOMY}

Favourable social and economic conditions constitute the essential framework for a stable development of savings.

Saving in the form of insurance becomes advantageous for the individual, and private insurance can thus extend its activity, when social attitudes and the economic situation favour the propensity to save.

If conditions change, the State can take over the coverage of risks through social insurance. By means of this institution, an anti-cyclical policy can be pursued : the amount of social security contributions, for instance, can be increased during the expansion of the cycle and the amounts thus accumulated can be used to grant benefits during the recession period, when contributions can be fixed at a lower percentage of wages.

Another type of policy can be pursued by government authorities : that of adjusting social security contributions to industrial profits, thereby directing the subsequent effects on economic growth.

Inflation can cause instability in decisional policies taken by private insurance companies. A solution to the unbalanced increase of costs can be found in index-linking. Life policies of this kind, for instance, can be closely related to investments in houses, to be bought by the insured themselves in price-linked instalments.

After a reference to present developments regarding risk instability and to possibilities of new forms of insurance, this paper considers the competition resulting from the opening of the EEC insurance markets as an opportunity for the Italian market to strengthen its structures.

Une étude sur l'importance économique de la prévoyance peut partir, fort à propos, de quelques considérations sur l'épargne qui en est l'expression primordiale.

Le souci de la sécurité est le ressort principal de l'épargne, celle-ci offrant une certitude raisonnable quant à la satisfaction des besoins futurs; mais la propension à économiser est également fonction de certaines conditions.

La première condition relève du milieu: si le contexte social n'offre aucune garantie de protection, le souci de l'épargne individuelle devient plus vif et pressant, alors que, dans un milieu nanti de sécurité sociale, ce besoin est évidemment moins prononcé. En outre, le sacrifice du moment présent se justifie pour autant qu'il corresponde à un profit futur de même ordre ; voilà donc une autre condition propre à encourager l'épargne : la stabilité du contexte économique et financier. Le contraire n'est pas moins évident : si l'érosion des valeurs monétaires est continuelle et consistante, c'est

\footnotetext{
* Professeur d'économie de l'assurance à l'université de Rome.
} 
la rentabilité de l'épargne individuelle qui est remise en question. Il est une autre condition qui tient, elle, aux mœurs : lorsque, à une époque donnée, la tradition ou l'attitude d'un groupe social ont tendance à exalter les valeurs du sacrifice, les individus qui le composent partagent généralement les conceptions et le comportement de leur propre collectivité et de leur temps. Une société axée sur la consommation de biens sous-estime, au contraire, le besoin d'épargne, aussi l'individu ne tarde-t-il guère à se conformer à la situation.

La propension individuelle à l'épargne subit, par conséquent, l'influence des conditions mentionnées qui, d'ailleurs, sont en corrélation entre elles. Si l'on restreint la consommation individuelle, on créera en même temps des ressources pour des investissements et pour un développement plus importants, sources de bien-être et de sécurité. $\mathrm{Si}$, au contraire, une partie considérable des revenus individuels est absorbée par la consommation, l'Etat devra intervenir pour former une épargne obligatoire, moyennant charges fiscales et sociales, au service de ce développement qui représente, lui, la garantie du lendemain. Mais, en l'espèce, la répartition des ressources se fera, pour la plupart, en dehors des choix individuels, ce qui reviendra à limiter le libre arbitre de chacun. Aussi le risque à courir est-il évident : celui de payer le développement par une atteinte à la liberté.

Il est une autre forme plus complexe d'épargne, fondée sur un besoin plus mûr de sécurité. Elle est réalisée par des collectivités homogènes dans le but de faire face à des risques communs, par l'intermédiaire d'institutions affectées à la collecte des primes : les entreprises privées d'assurances. L'épargne est, dans ce cas, solidaire et spontanée; aussi est-elle le propre de groupements relativement restreints. Dans cette catégorie de l'épargne également, si la collectivité assurée s'élargit et que ses caractéristiques se perdent dans une plus vaste hétérogénéité, l'Etat devra intervenir (par le biais des assurances sociales) pour rendre obligatoire cette participation individuelle au sacrifice que le simple jeu de la rentabilité n'est plus en mesure de garantir.

Quelle que soit la forme qu'elle révèle, l'épargne a un coût; ce coût est dû à la médiation. Ce coût de collecte n'est pas un élément exclusif et distinctif de l'épargne d'assurance : il s'agit, de toute façon, d'une opération coûteuse. Le coût de la médiation financière, réalisée par des entreprises d'assurances ou des organismes de sécurité sociale, par des sociétés d'assurance mutuelle ou des établissements de crédits, est toujours assez élevé. En toute vraissemblance, il est destiné à augmenter, un facteur typique et d'actualité de ce coût étant représenté par l'utilisation de services d'une technicité plus évoluée.

De par ses effets économiques, la politique des investissements prend une importance considérable, même si les réserves mathématiques des entreprises d'assurances ne sont pas énormes et si la durée moyenne de l'épargne réalisée par les organismes de sécurité sociale est brève. En Italie, en effet, la masse financière des charges sociales n'est pas dirigée actuellement vers l'investissement, mais transformée en prestations immédiates, elle est directement projetée dans la consommation: le système financier de la répartition, aujourd'hui en usage, ne permet pas de créer de disponibilités monétaires, si ce n'est pour de courtes périodes, ni ne prévoit de formation de capital. Néanmoins, si limitée soit-elle, cette masse financière peut représenter un instrument de politique économique. Il pourrait être avantageux de constituer de petites réserves, moyennant une augmentation des charges sociales dans les périodes de conjoncture 
favorable; ces réserves "anticycliques 》 seraient utilisées dans les phases de récession, et serviraient à canaliser d'une manière plus souple et plus supportable la poussée que les charges exercent sur les coûts de l'entreprise ${ }^{1}$. L'application de la règle de proportionnalité des charges sociales par rapport à des paramètres autres que le salaire, tel que, par exemple, le revenu de l'entreprise, aurait de remarquables effets induits susceptibles d'être "dirigés » à l'instar d'actes de politique économique. Un mécanisme de ce genre pourrait être mis au service d'une politique industrielle pour répartir différemment sur les divers secteurs la charge sociale du coût du travail: il pourrait s'ensuivre des avantages dans certains secteurs en crise.

Grâce à une vivace politique sociale, la sécurité s'est accrue, en Italie, pour la plupart des travailleurs salariés, dont la rémunération en valeur réelle n'a pas fléchi durant la dernière décennie, alors que leur sécurité sociale a enregistré de sensibles améliorations. Aussi faut-il noter, à ce propos, l'importante redistribution du revenu que la sécurité sociale a provoquée dans notre pays parmi les différents secteurs de la production, de même qu'entre les différentes régions.

L'entrepreneur, de son côté, trouve une plus grande sécurité dans les sociétés d'assurances privées et publiques. L'assurance privée soutient, en effet, l'initiative industrielle et pare à ses risques en la garantissant en cas d'événements dommageables pouvant entraîner l'exclusion de l'entreprise du marché (la réalisation, par exemple, d'un "gros risque »), alors que l'assurance sociale intervient pour protéger, par des formes accentuées de solidarité, le niveau de l'emploi dans les secteurs en crise, par exemple par l'octroi de certaines prestations, comme celles de la caisse de compensation des salaires, protégeant conjointement l'entrepreneur et le salarié, ou celles de la retraite anticipée.

Si la garantie de l'assurance privée encourage et stimule l'initiative industrielle en tendant un filet de sauvetage en cas de réalisation d'un sinistre, la sécurité sociale remplit elle aussi un rôle de soutien de l'économie, dans la mesure où elle intervient, avec ses prestations, pour stimuler la demande (tout au moins celle de la consommation des ménages) en évitant ainsi des diminutions importantes dans la consommation de biens et de services primaires ou de certains bien durables.

L'économie, dans son ensemble, profite de l'assurance : que l'on songe, notamment, à la marge de manœuvre qu'offre l'épargne de la sécurité sociale. Sur la formation de cette épargne agit également, en Italie, et d'une manière prépondérante, le phénomène inflationniste : la dévaluation a entraîné, en son temps, le choix du système "de répartition » dans les assurances sociales, aussi est-ce l'instabilité monétaire qui pousse l'assuré à opter pour des types de police à réserve mathématique minimum. C'est également par rapport au phénomène inflationniste que l'on jauge l'entreprise d'assurances, lorsqu'elle doit faire face à la montée des frais administratifs, parce que les dépenses de gestion se conforment aussitôt à la hausse du coût de la vie, et qu'elle doit alors redoubler ses efforts pour augmenter le portefeuille. C'est là un fait qu'il y a lieu de souligner: l'inflation ne frappe pas uniquement l'épargnant, mais aussi l'entreprise d'assurances.

1 Un effet anticyclique de la sécurité sociale serait utile, à la condition de ne pas sacrifier les objectifs fondamentaux, qui sont des objectifs de politique sociale - C.E.E. - Etudes : série «POLITIQUE SOCIALE » 1970 - No 21. 
Dans la branche assurance sur la vie, l'inflation a des répercussions considérables sur les revenus des réserves. Les valeurs d'Etat et les obligations subissent entièrement le contrecoup de la dévaluation; mais l'entreprise ne peut se soustraire aux investissements que la loi impose. Les investissements dans l'immobilier (autre orientation suggérée par les autorités de tutelle) déclenchent, en période d'inflation, une baisse du revenu réel. L'incapacité de l'investissement immobilier de suivre le glissement monétaire dépend de causes multiples : en premier lieu les lois concernant les loyers, mais également la revalorisation des immeubles qui, à juste titre, figure au bilan comme plus-value, et concourt à diminuer ultérieurement le rendement, alors qu'elle vient accroître l'imposition fiscale. En dépit de ces difficultés, les investissement immobiliers absorbent à peu près la moitié des disponibilités des compagnies d'assurances, alors que les rendements les plus élevés proviennent des dépôts bancaires à vue.

Dans la recherche de formes d'assurance permettant de faire face à la dévaluation, une solution satisfaisante peut être trouvée dans les formules d'indexation. Au cours des années 70, le marché des assurances sur la vie a été fortement dominé par les polices rajustables qui, toutefois, limitent la garantie à un pourcentage excessivement faible de réajustement: il serait plus judicieux de réagir contre l'inflation en utilisant des paramètres mieux aptes à asseoir la prime et les réserves sur des valeurs représentatives ${ }^{2}$.

Dans les branches de base, l'inflation se traduit également par de complexes phénomènes de déséquilibre dans le rapport primes/indemnisations : un raccourcissement des délais d'indemnisation s'est parfois avéré utile en exerçant une influence positive sur l'efficacité du service fourni.

Un autre phénomène encore caractérise l'évolution actuelle des assurances : l'apparition de nouveaux risques. La sécurité va constamment de pair avec le développement industriel et celui de la société, aussi, parallèlement au progrès, emprunte-t-elle de nouvelles formes et acquiert-elle de nouvelles dimensions.

Dans les assurances privées, l'apparition de ces risques nouveaux issus des technologies inédites mises en œuvre élargit la dimension du concept d'assurabilité, tandis que, dans le secteur de la production, l'échelle industrielle s'agrandit et l'entreprise, fidèle au mythe de l'expansion continuelle, franchit la limite de la dimension optimale et s'expose à une baisse de ses rendements.

Mais, fréquemment, les nouvelles technologies sont appliquées dans des contextes économiques vagues, de sorte qu'il est difficile d'évaluer les conséquences des nouveaux

2 Un exemple : comme dans la réalité italienne, le niveau des salaires est revalorisé en permanence et parallèlement à la hausse du coût de la vie, la prime d'assurance pourrait être liée à une indemnité-salaire variant en fonction de cette hausse. Simultanément, on pourrait aligner sur le même paramètre les réserves concrétisées en biens - tels que, par exemple, des logements pour les travailleurs salariés - qui seraient payés aux bénéficiaires par des versements proportionnels à cette indemnité variable. Il s'agirait, pour l'assureurinvestisseur, d'une recette indexée sur la valeur de la monnaie (et l'on eviterait les rendements actuels décroissants, auxquels correspond l'enrichissement de ceux qui payent en versements constants le bien logement); quant à l'assureur, son profit pourrait résider dans le fait qu'il serait le bénéficiaire d'un investissement socialement utile et rémunérateur. 
risques éventuels qu'elles comportent ; par conséquent, l'appréciation même du risque devient de plus en plus problématique et le concept même d'assurabilité n'est pas sans subir de sensibles variations.

L'objectivation du risque est vraisemblablement un des éléments clés du marché actuel des assurances. A ce propos, la couverture des risques nouveaux pourrait trouver une solution satisfaisante du point de vue technique dans la concentration de la garantie en ce que l'on pourrait définir comme "le corps central » du risque, en laissant à la charge de l'assuré le découvert initial et à celle de l'Etat la partie finale au sens du terme catastrophe.

Pour ce qui est des risques plus répandus, on s'est acheminé vers des formes de socialisation : la création d'un fonds commun dans les assurances automobiles n'est pas sans représenter déjà une forme de sauvegarde collective; il est alimenté par les entreprises, mais au moyen de prélèvements sur les primes des personnes assurées.

En ce qui concerne la branche de la responsabilité civile, la demande tend de plus en plus vers une protection globale de l'individu par l'intermédiaire de la compagnie. Cette évolution régulière dans la qualité de la demande d'assurances pose, en outre, le problème du réajustement des structures afin d'y répondre par une offre plus satisfaisante pour la clientèle.

Dans le domaine de l'assurance-vie, les organismes d'assurances font vigoureusement obstacle à la modernisation du service assurances en se refusant à en modifier les bases techniques. Je songe aux tables d'élimination - qu'une loi récente vient d'ailleurs de rajeunir - mais surtout au taux d'intérêt adopté pour calculer le tarif. Il ne suffit pas de majorer ce taux théorique d'un pourcent tous les dix ans pour rendre l'assurance-vie plus intéressante pour l'assuré, dans un contexte inflationniste ayant largement démontré qu'il ne veut plus se maintenir désormais aux 3 ou $4 \%$ des heureux temps de la dévaluation rampante. On ne propose point ici d'élever le taux au niveau de l'inflation ou à celui des dépôts bancaires (répondant à des durées différentes d'investissement), mais il est sans doute possible, voire utile, de ménager plus de latitude à la technique de l'indexation des primes et des réserves.

L'assureur devra faire preuve de plus de circonspection encore dans le maniement des capitaux à investir ; néanmoins, l'aléa d'une nouvelle conception des investissements (et des réinvestissements) est le tribut qu'il faudra payer si l'on veut répandre plus largement les formes de prévoyance spontanée. On vise tout particulièrement ces formes collectives offrant un soutien supplémentaire à de vastes catégories de particuliers dont la subsistance, à l'âge de la retraite, est désormais du ressort des assurances sociales, coûteuses pour la collectivité si elles sont élevées à des niveaux très proches des salaires réels.

On ne saurait oublier, à l'issue de ces considérations, l'importance de plus en plus grande que prend la dimension de l'entreprise d'assurances. Par suite de l'intégration du marché des assurances, l'entreprise italienne en vient à se mesurer de plus près avec celles de la Communauté européenne; de cette confrontation avec la concurrence, découlent tant la nécessité de mettre au point ses propres services pour mieux répondre aux exigences d'un marché plus vaste et plus "sophistiqué 》, que l'urgence de renforcer ses structures opérationnelles et financières. 
C'est dans ce nouveau contexte que le rôle de l'entreprise de réassurances gagne en importance, face aux risques nouveaux, plus graves, plus complexes et plus nombreux. Par besoin de sécurité, le transfert des risques d'une entreprise à une autre - moyennant rétrocession - institue tout un réseau de liaisons entre sociétés de pays différents et aboutit ainsi à la création d'un vaste filet de sauvegarde garantissant au mieux la sécurité de chacune d'entre elles. 